\title{
Characterizing the risk and outcome profiles of lumbar fusion procedures in patients with opioid use disorders: a step toward improving enhanced recovery protocols for a unique patient population
}

\author{
Michael L. Martini, BA, Dominic A. Nistal, BA, Brian C. Deutsch, BS, and John M. Caridi, MD \\ Department of Neurosurgery, Icahn School of Medicine at Mount Sinai, New York, New York
}

OBJECTIVE The authors set out to conduct the first national-level study assessing the risks and outcomes for different lumbar fusion procedures in patients with opioid use disorders (OUDs) to help guide the future development of targeted enhanced recovery after surgery (ERAS) protocols for this unique population.

METHODS Data for patients with or without OUDs who underwent an anterior lumbar interbody fusion (ALIF), posterior lumbar interbody fusion (PLIF), or lateral transverse lumbar interbody fusion (LLIF) for lumbar disc degeneration (LDD) were collected from the 2013-2014 National (Nationwide) Inpatient Sample database. Multivariable logistic regression was implemented to analyze how OUD status impacted in-hospital complications, length of hospital stay, discharge disposition, and total charges by procedure type.

RESULTS A total of 139,995 patients with LDD were identified, with 1280 patients $(0.91 \%)$ also having a concurrent OUD diagnosis. Overall complication rates were higher in OUD patients ( $48.44 \%$ vs $31.01 \%, p<0.0001)$. OUD patients had higher odds of pulmonary $(p=0.0006)$, infectious $(p<0.0001)$, and hematological $(p=0.0009)$ complications. Multivariate regression modeling of outcomes by procedure type showed that after ALIF, OUD patients had higher odds of nonhome discharge $(p=0.0007)$, extended hospitalization $(p=0.0002)$, and greater total charges $(p=0.0054)$. This analysis also revealed that OUD patients faced higher odds of complication $(p=0.0149$ and $p=0.0471)$, extended hospitalization $(p=0.0439$ and $p=0.0001)$, and higher total charges $(p<0.0001$ and $p<0.0001)$ after PLIF and LLIF procedures, respectively.

CONCLUSIONS Obtaining a better understanding of the risks and outcomes that OUD patients face perioperatively is a necessary step toward developing more effective ERAS protocols for this vulnerable population. This study, which sought to characterize the outcome profiles for lumbar fusion procedures in OUD patients on a national level, found that this population tended to experience increased odds of complications, extended hospitalization, nonhome discharge, and higher total costs. Results from this study warrant future prospective studies to better the understanding of these associations and to further the development of better ERAS programs that may improve patient care and reduce cost burden.

https://thejns.org/doi/abs/10.3171/2019.1.FOCUS18652

KEYWORDS opioid use disorders; lumbar disc degeneration; enhanced recovery; spine surgery; National Inpatient Sample

$\mathrm{R}$ ECOVERY after spine surgery can be a complex process involving postoperative pain, mobilization, return to work, quality of life, and overall patient outcome. One of the challenges of recovery after surgery is the large dependence that both providers and patients have for opioid analgesics. Recently, providers have been moving toward enhanced recovery after surgery (ERAS) multimodal systems that emphasize the importance of a multidisciplinary approach, improvement in outcomes, and reduction in cost. ERAS emphasizes the reduction of

ABBREVIATIONS ALIF = anterior lumbar interbody fusion; APR-DRG = All Patient Refined Diagnosis-Related Groups; ERAS = enhanced recovery after surgery; HCUP = Healthcare Cost and Utilization Project; ICD-9-CM = International Classification of Diseases, Ninth Edition, Clinical Modification; LLIF = lateral transverse lumbar interbody fusion; LDD = lumbar disc degeneration; NIS = National (Nationwide) Inpatient Sample; OUD = opioid use disorder; PLIF = posterior lumbar interbody fusion. ACCOMPANYING EDITORIAL DOI: 10.3171/2019.1.FOCUS1944.

SUBMITTED November 28, 2018. ACCEPTED January 14, 2019.

INCLUDE WHEN CITING DOI: 10.3171/2019.1.FOCUS18652. 
opioid analgesics for a number of reasons, including reduction in opioid dependence, reduced length of stay, improved postoperative pain, and reduction in postoperative ileus. ${ }^{17}$

Since the founding of the ERAS society in the early 2000s, numerous studies have been published with efforts to improve postoperative outcomes by reducing opioid analgesic use. The development of new pain medication regimens and recovery pathways found a reduction in postoperative opioid use, earlier ambulation, and lower instances of nausea, sedation, and dizziness. ${ }^{9,22,35}$ Preoperative chronic opioid use has a clear influence on postoperative outcomes and is a growing problem that must be addressed when developing new ERAS systems.

Patients with opioid use disorders (OUDs) display problematic patterns of opioid consumption and represent a population that is particularly susceptible to opioid misuse after discharge. Patients with OUDs require additional considerations in preoperative planning and in developing new ERAS protocols for effective management in the perioperative period, which must inherently account for the unique risks and adverse outcomes faced by this population during their hospitalizations. Unfortunately, the perioperative period is a very vulnerable time for OUD patients, and there is a dearth of knowledge pertaining to the specific risks and outcomes they face during this time. ${ }^{36}$ This has, to an extent, limited the ability of ERAS protocols to provide optimal care for this patient population.

Increased preoperative opioid consumption is associated with worse patient-reported outcomes in cervical, thoracolumbar, and lumbar spine surgery to treat structural disease. ${ }^{16}$ Increased opioid dependence, morbidity, and mortality in patients with preoperative opioid use was found across a number of studies for both spine and orthopedic procedures. ${ }^{1,24,37}$ For several years, the use of surgical fusion procedures to treat lumbar stenosis and degenerative disease has been steadily increasing. ${ }^{18,19}$ These procedures are performed anteriorly (anterior lumbar interbody fusion [ALIF]), posteriorly (posterior lumbar interbody fusion [PLIF]), laterally (lateral transverse lumbar interbody fusion [LLIF]), and transforaminally (transforaminal lumbar interbody fusion). While previous studies have examined the influence of preoperative chronic opioid use on the complications and outcomes in PLIF procedures,${ }^{14}$ none have comprehensively assessed and compared the influence of OUDs on outcomes for various approaches of lumbar fusion procedures. Furthermore, no study has determined outcome profiles for these procedures on a national level for the purpose of influencing new enhanced recovery protocols in spine surgery for patients with OUDs.

\section{Methods}

\section{Data Source}

Data from 2013 to 2014 were obtained from the National (Nationwide) Inpatient Sample (NIS) of the Healthcare Cost and Utilization Project (HCUP), Agency for Healthcare Research and Quality. The NIS has data for over 7 million hospitalizations per year, making it the largest all-payer national database. The NIS consists of a stratified sample of $20 \%$ of nonfederal hospitals in the United
States. To obtain regional and national estimates, an encoded weighting variable was applied that accounts for the stratified clustered sampling of the database, as specified by HCUP (https://www.hcup-us.ahrq.gov/nisoverview. jsp). Our institutional review board has exempted studies involving the NIS from individual review.

\section{Inclusion Criteria and Patient Stratification}

Patients were included if they had an International Classification of Diseases, Ninth Edition, Clinical Modification (ICD-9-CM) diagnosis code of lumbar disc degeneration (LDD; ICD-9-CM code 722.52). Patients were included in the OUD population if they had a diagnosis code for opioid dependence (304.00-304.03, 304.70-304.73) or nondependent opioid abuse (305.50-305.53) along with the code for LDD. In some analyses, patients were stratified based on the procedure type performed for lumbar spinal correction. This was done using procedure codes for primary or repeat ALIF (81.06, 81.36), PLIF (81.07, 81.37), or LLIF $(81.08,81.38)$.

\section{Covariates}

Data were obtained on patients' age, sex, race, hospital division, admission type, comorbidities, primary payer status, discharge status, median income by ZIP code, and All Patient Refined Diagnosis-Related Groups (APRDRG) scores for severity of illness and mortality risk. The treatment modality employed to treat LDD was also considered as a covariate, particularly when assessing overall complication rates.

\section{Outcomes}

Primary outcomes included in-hospital mortality rate, overall complication rate, complication rate by organ system, and certain pertinent perioperative complications. Secondary outcome measures included discharge disposition, mean length of hospital stay, and total charges for the hospital visit. Any discharge that was not to home was considered a nonhome discharge. Hospitalization lengths greater than the 75th percentile for the entire study population were considered to be extended hospitalizations.

Organ system complications were determined for cardiac (410.xx or 785.xx), pulmonary $(514,518 . x x$, or 512.xx), neurological (stroke 433.x or 434.x, transient cerebral ischemia 435.x, and seizures 345.xx, and neurological complications after procedure 997.01 or 997.09), infectious (041.xx, 38.xx, 320.xx, 324.1, 481-486, 507.0, 595.0, 790.7, 995.9x, 996.64, 997.31, 998.59, 999.31), renal (584.x), hematological (285.xx or 998.1x, or red blood cell transfusion 99.04), gastrointestinal (578.x, 560.1, or 00845), and peripheral vascular (venous thromboembolic 453.xx or 415.xx) systems. Sodium disturbance events $(253.5,253.6,276.0$, or 276.1) as well as the proportion of patients who also underwent a gastrojejunostomy (43.1, $43.11,43.19$, or 46.32$)$ or tracheostomy $(31.1,31.2,31.21$, or 31.29) were also examined.

\section{Statistical Analysis}

Statistical analysis was conducted using SAS (version 9.4, SAS Institute), wherein the survey procedures were 
implemented to account for the special survey design of the NIS. The NIS now comprises a sampling of all discharges from HCUP-participating hospitals in the country rather than a sampling of hospitals from which all discharge records were obtained. Thus, each patient discharge was used as the sampling unit, discharge weight used as the sampling-weight variable, and NIS stratum as the stratum. Chi-square tests analyzed categorical baseline characteristics between the 2 populations. Fisher's exact test was used for contingency tables with expected counts less than 1 under the null hypothesis of independence. The means of any continuous variables between the 2 groups were assessed with a 2-sided, 2-sample t-test. Individual primary and secondary outcomes based on OUD status were first examined using univariate regression models. Multivariable regression models were later constructed using predictor variables previously identified as differing significantly between patients in the 2 cohorts as covariates. A Morel adjustment was applied to logistic regression analyses pertaining to rare conditions to reduce any small sample bias that may exist. A p value $<0.05$ was set a priori as a threshold for statistical significance. All figures were produced using Prism 7 (GraphPad).

\section{Results \\ Demographics}

A weighted total of 139,995 patients undergoing an ALIF, PLIF, or LLIF procedure for LDD were included in this study, of whom 1280 patients $(0.91 \%)$ also carried a concurrent OUD diagnosis. Table 1 displays the baseline characteristics of the patient populations based on OUD status. Of note, the patient populations differed significantly in their distributions for age $(\mathrm{p}<0.0001)$, primary insurance payer ( $\mathrm{p}<0.0001)$, median income by ZIP code $(\mathrm{p}=0.0002)$, discharge status $(\mathrm{p}=0.0008)$, and hospital geographic division $(\mathrm{p}<0.0001$; Table 1$)$. LDD patients with OUDs also had a significantly greater number of diagnoses on record $(\mathrm{p}<0.0001)$, number of procedures on record $(\mathrm{p}=0.0003)$, number of chronic conditions ( $\mathrm{p}$ $<0.0001)$, mean APR-DRG risk of mortality scores ( $\mathrm{p}=$ 0.0065 ), and mean APR-DRG severity of illness scores ( $\mathrm{p}$ $<0.0001$; Table 2). This patient population also had significantly higher frequencies of certain comorbidities, including alcohol abuse $(\mathrm{p}<0.0001)$, deficiency anemia ( $\mathrm{p}$ $=0.0063)$, arthritis $(\mathrm{p}=0.0024)$, anemia due to chronic blood loss $(p=0.0198)$, chronic lung disease $(p=0.0003)$, depression $(\mathrm{p}<0.0001)$, fluid and electrolyte disorders ( $\mathrm{p}$ $=0.0049)$, obesity $(\mathrm{p}=0.0424)$, peripheral vascular disease $(\mathrm{p}=0.0341)$, psychosis $(\mathrm{p}<0.0001)$, weight loss $(\mathrm{p}<$ $0.0001)$, and other neurological disorders ( $p=0.0040$; Fig. 1). Of note, the LDD population with OUD had a significantly lower rate of hypertension $(\mathrm{p}=0.0080)$.

\section{Outcomes}

Despite the higher mean APR-DRG risk of mortality scores in the group with OUDs, none of these patients experienced an in-hospital mortality after their procedure. Patients with concurrent OUDs did, however, experience significantly higher overall rates of complication (48.44\%) than those without OUDs $(31.01 \%)(\mathrm{p}<0.0001)$. Univari-
TABLE 1. Demographics of the study population by OUD status

\begin{tabular}{|c|c|c|c|}
\hline & OUD St & atus* & \\
\hline & $\begin{array}{c}\text { LDD } \\
(n=138,715)\end{array}$ & $\begin{array}{l}\text { LDD + OUD } \\
(n=1280)\end{array}$ & $\begin{array}{c}\mathrm{p} \\
\text { Value }\end{array}$ \\
\hline Age, yrs & & & $<0.0001$ \\
\hline $0-44$ & $27,565(20.1)$ & $325(25.5)$ & \\
\hline $45-64$ & $65,630(48.0)$ & $745(58.4)$ & \\
\hline $65-74$ & $32,790(24.0)$ & $175(13.7)$ & \\
\hline$\geq 75$ & $10,810(7.9)$ & $30(2.4)$ & \\
\hline Sex & & & 0.2282 \\
\hline Male & $63,725(45.96)$ & $540(42.19)$ & \\
\hline Female & $74,935(54.04)$ & $740(57.81)$ & \\
\hline Race & & & 0.8210 \\
\hline White & $109,955(84.14)$ & $850(84.58)$ & \\
\hline Black & $9555(7.31)$ & $65(6.47)$ & \\
\hline Hispanic & $6225(4.76)$ & $100(4.98)$ & \\
\hline Asian & $635(0.49)$ & $5(0.50)$ & \\
\hline Other & $3900(4.00)$ & $35(3.48)$ & \\
\hline Insurance & & & $<0.0001$ \\
\hline Medicare & $53,355(38.52)$ & $530(41.41)$ & \\
\hline Medicaid & $9040(6.53)$ & $170(13.28)$ & \\
\hline Private & $61,110(44.12)$ & $465(36.33)$ & \\
\hline Other & $15,000(10.83)$ & $115(4.95)$ & \\
\hline Median income by ZIP code & & & 0.0002 \\
\hline 0-25th percentile & $31,700(23.29)$ & $215(17.06)$ & \\
\hline 26-50th percentile & $39,345(28.91)$ & $295(23.41)$ & \\
\hline 51-75th percentile & $35,650(26.19)$ & $340(26.98)$ & \\
\hline 76-100th percentile & $29,415(21.61)$ & $410(32.54)$ & \\
\hline Discharge status & & & 0.0008 \\
\hline Home (routine) & $117,035(84.4)$ & $955(74.6)$ & \\
\hline Short-term care & $470(0.3)$ & $5(0.4)$ & \\
\hline Skilled nursing facility & $25(0)$ & $0(0)$ & \\
\hline Died in hospital & $95(0.1)$ & $0(0)$ & \\
\hline $\begin{array}{l}\text { Designated cancer } \\
\text { center or other }\end{array}$ & $21,090(15.2)$ & $320(25.0)$ & \\
\hline Hospital division & & & $<0.0001$ \\
\hline New England & $4865(3.5)$ & $60(4.7)$ & \\
\hline Middle Atlantic & $14,465(10.5)$ & $120(9.4)$ & \\
\hline East North Central & $23,550(17.0)$ & $165(12.9)$ & \\
\hline West North Central & $10,145(7.3)$ & $265(20.7)$ & \\
\hline South Atlantic & $30,070(21.7)$ & $240(18.8)$ & \\
\hline East South Central & $11,155(8.0)$ & $30(2.3)$ & \\
\hline West South Central & $20,405(14.7)$ & $45(3.5)$ & \\
\hline Mountain & $15,160(10.9)$ & $190(14.8)$ & \\
\hline Pacific & $8900(6.4)$ & $165(12.9)$ & \\
\hline Admission type & & & 0.1534 \\
\hline Elective & $130,220(94.3)$ & $1180(92.2)$ & \\
\hline $\begin{array}{l}\text { Nonelective (urgent or } \\
\text { emergency) }\end{array}$ & $7919(5.7)$ & $100(7.8)$ & \\
\hline
\end{tabular}

CONTINUED ON PAGE 4 » 
» CONTINUED FROM PAGE 3

TABLE 1. Demographics of the study population by OUD status

\begin{tabular}{cccc}
\hline & \multicolumn{2}{c}{ OUD Status $^{*}$} & \\
\cline { 2 - 3 } & LDD & LDD + OUD & $p$ \\
$(n=138,715)$ & $(n=1280)$ & Value \\
\hline $\begin{array}{c}\text { APR-DRG risk of mortality } \\
\text { score }\end{array}$ & & & 0.0014 \\
\hline $\begin{array}{c}\text { Minor likelihood of } \\
\text { dying }\end{array}$ & $119,525(86.2)$ & $1000(78.1)$ & \\
\hline $\begin{array}{c}\text { Moderate likelihood of } \\
\text { dying }\end{array}$ & $14,695(10.6)$ & $190(14.8)$ & \\
\hline $\begin{array}{c}\text { Major likelihood of } \\
\text { dying }\end{array}$ & $3650(2.6)$ & $70(5.5)$ & \\
\hline $\begin{array}{c}\text { Extreme likelihood of } \\
\text { dying }\end{array}$ & $840(0.6)$ & $20(1.6)$ & \\
\hline $\begin{array}{c}\text { APR-DRG severity of } \\
\text { illness score }\end{array}$ & & & \\
\hline Minor loss of function & $73,410(52.9)$ & $225(17.6)$ & \\
\hline $\begin{array}{c}\text { Moderate loss of } \\
\text { function }\end{array}$ & $54,155(39.0)$ & $795(62.1)$ & \\
\hline Major loss of function & $10,335(7.5)$ & $220(17.2)$ & \\
\hline $\begin{array}{c}\text { Extreme loss of } \\
\text { function }\end{array}$ & $810(0.6)$ & $40(3.1)$ \\
\hline
\end{tabular}

$p<0.05$ was used as a threshold for statistical significance.

${ }^{*}$ Values given as weighted count (\%). ate regression revealed that patients with OUDs faced significantly higher odds of complications after all 3 of the procedure types examined, including ALIFs (OR 2.707, 95\% CI 1.725-4.248; $p<0.0001$ ), PLIFs (OR 2.251, 95\% CI $1.392-3.641 ; \mathrm{p}=0.0009$ ), and LLIFs (OR 1.696, 95\% CI 1.165-2.468; $\mathrm{p}=0.0059$ ) (Table 3).

Univariate regressions were applied to a selected set of pertinent complications related to specific organ systems (Fig. 2). Patients with OUDs faced significantly increased odds of complication in certain organ systems, including pulmonary complications (OR 2.295, 95\% CI 1.425 3.696; $\mathrm{p}=0.0006$ ), infectious complications (OR 3.716, 95\% CI 2.097-6.584; $\mathrm{p}<0.0001$ ), and hematological complications (OR 1.648, 95\% CI 1.226-2.215; $\mathrm{p}=0.0009)$. Of note, patients with OUDs did not experience significantly higher odds of neurological complications (OR 2.015, 95\% CI 0.989-4.106; $\mathrm{p}=0.0537$ ).

We examined several specific complications with known relevance in spinal fusion procedures to more thoroughly examine the unique complication profiles of patients with OUDs after these procedures (Table 4). We found that patients with OUDs had significantly higher odds of pneumonia (OR 3.059, 95\% CI 1.285-7.283; $\mathrm{p}=0.0115$ ), venous thromboembolism (OR 4.165, 95\% CI 1.851-9.372; p = 0.0006 ), pulmonary embolism (OR $4.973,95 \%$ CI 1.499 16.500; $\mathrm{p}<0.0088$ ), postoperative anemia (OR 1.957, 95\% CI 1.406-2.722; $\mathrm{p}<0.0001$ ), wound infection (OR 9.387, 95\% CI 3.357-26.249; $\mathrm{p}<0.0001$ ), systemic inflammatory response syndrome (OR 9.664, 95\% CI 2.846-32.810; $\mathrm{p}<0.0001)$, urinary tract infection (OR 2.626, 95\% CI $1.407-4.901 ; \mathrm{p}=0.0024)$, or requiring a tracheostomy (OR $18.130,95 \%$ CI $2.130-154.300 ; \mathrm{p}=0.0080$ ).

TABLE 2. Differences in baseline characteristics in the study population by OUD status

\begin{tabular}{|c|c|c|c|}
\hline & \multicolumn{2}{|c|}{ OUD Status } & \multirow{2}{*}{$\begin{array}{c}p \\
\text { Value }\end{array}$} \\
\hline & $\operatorname{LDD}(n=138,715)$ & LDD + OUD $(n=1280)$ & \\
\hline \multicolumn{4}{|c|}{ APR-DRG risk of mortality } \\
\hline Mean $(95 \% \mathrm{Cl})$ & $1.18(1.17-1.18)$ & $1.305(1.21-1.40)$ & 0.0065 \\
\hline SEM & 0.004 & 0.046 & \\
\hline \multicolumn{4}{|c|}{ APR-DRG severity of illness } \\
\hline Mean $(95 \% \mathrm{Cl})$ & $1.56(1.54-1.57)$ & $2.06(1.97-2.15)$ & $<0.0001$ \\
\hline SEM & 0.007 & 0.045 & \\
\hline \multicolumn{4}{|l|}{ Age, yrs } \\
\hline Mean $(95 \% \mathrm{Cl})$ & $56.8(56.6-57.1)$ & $52.7(51.4-54.0)$ & $<0.0001$ \\
\hline SEM & 0.127 & 0.679 & \\
\hline \multicolumn{4}{|c|}{ No. of diagnoses on record } \\
\hline Mean $(95 \% \mathrm{Cl})$ & $9.00(8.87-9.12)$ & $13.32(12.48-14.16)$ & $<0.0001$ \\
\hline SEM & 0.064 & 0.429 & \\
\hline \multicolumn{4}{|c|}{ No. of chronic conditions on record } \\
\hline Mean $(95 \% \mathrm{Cl})$ & $4.95(4.89-5.01)$ & $7.43(7.04-7.83)$ & $<0.0001$ \\
\hline SEM & 0.031 & 0.202 & \\
\hline \multicolumn{4}{|c|}{ No. of procedures on record } \\
\hline Mean $(95 \% \mathrm{Cl})$ & $5.80(5.73-5.88)$ & $6.40(6.08-6.71)$ & 0.0003 \\
\hline SEM & 0.037 & 0.161 & \\
\hline
\end{tabular}

$p<0.05$ was used as a threshold for statistical significance. 


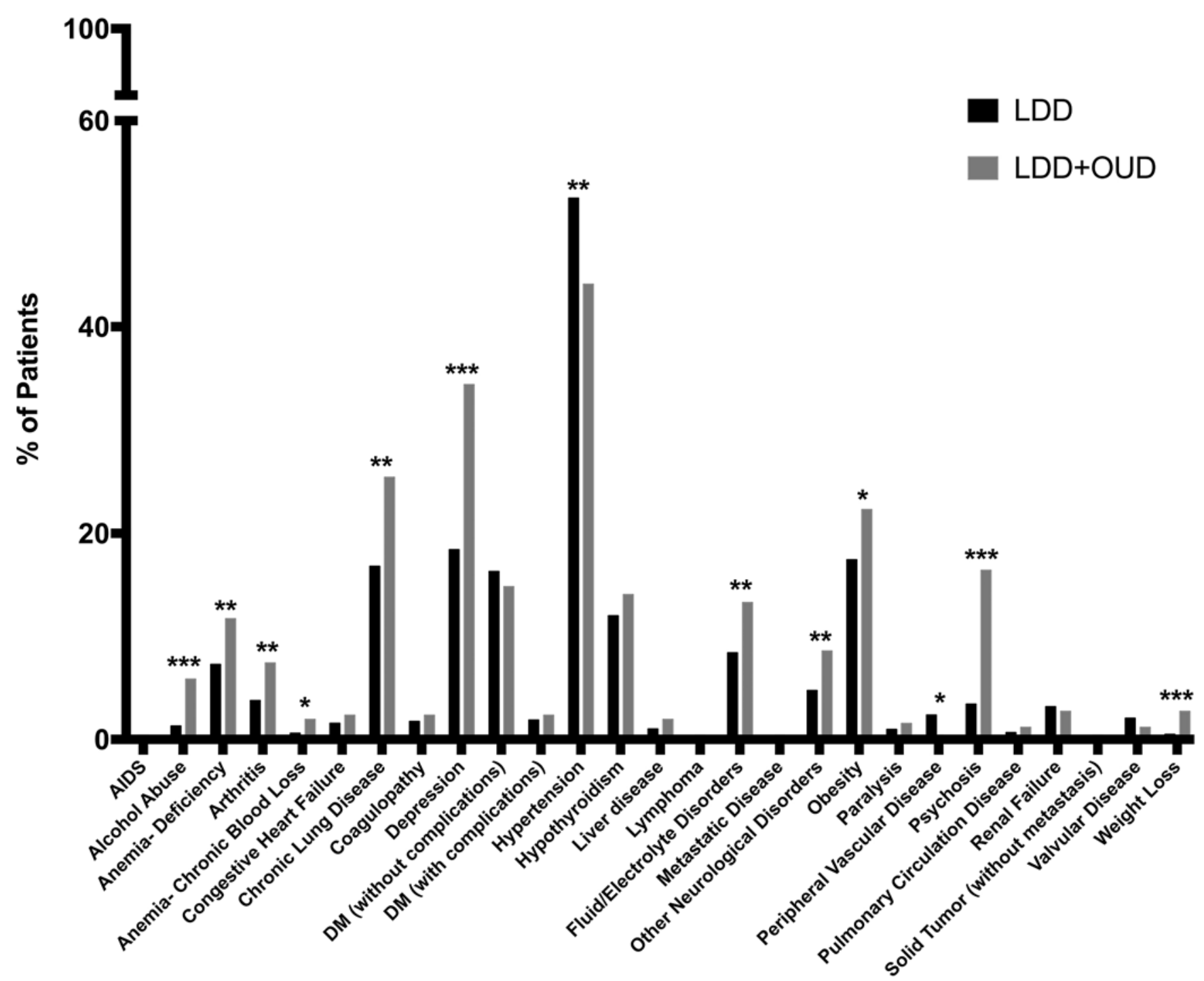

FIG. 1. Comorbidity landscape of patients undergoing lumbar fusion procedures by OUD status. A p value $<0.05$ was used as a threshold for establishing statistical significance. ${ }^{*} p<0.05 ;{ }^{* *} p<0.01 ;{ }^{* * *} p<0.0001$. DM $=$ diabetes mellitus.

Table 5 displays the results of univariate regression analysis examining the impact of OUDs on secondary outcome measures for resource utilization following fu- sion procedures for lumbar disc degeneration. We found that LDD patients with concurrent OUDs experienced significantly longer mean hospitalization lengths of stay ( $p$

TABLE 3. Univariate regression models evaluating the impact of OUD status on in-hospital mortality and complications by procedure type

\begin{tabular}{|c|c|c|c|c|}
\hline & \multicolumn{2}{|c|}{ OUD Status* } & \multirow[b]{2}{*}{ OR $(95 \% \mathrm{Cl})$} & \multirow[b]{2}{*}{ p Value } \\
\hline & LDD & LDD + OUD & & \\
\hline In-hospital mortality rate & $95 / 138,715(0.10)$ & $0 / 1280(0)$ & NA & NA \\
\hline All complications & $43,010 / 138,715(31.01)$ & $620 / 1280(48.44)$ & $2.090(1.640-2.665)$ & $<0.0001$ \\
\hline ALIF complications & $11,205 / 35,790(31.15)$ & $190 / 345(55.07)$ & 2.707 (1.725-4.248) & $<0.0001$ \\
\hline PLIF complications & $12,770 / 44,130(28.94)$ & $165 / 345(47.83)$ & 2.251 (1.392-3.641) & 0.0009 \\
\hline LLIF complications & $19,035 / 58,615(32.47)$ & $265 / 590(45.00)$ & $1.696(1.165-2.468)$ & 0.0059 \\
\hline
\end{tabular}

$\mathrm{NA}=$ not applicable.

$p<0.05$ was used as a threshold for statistical significance.

*Values given as weighted count/total (\%). 


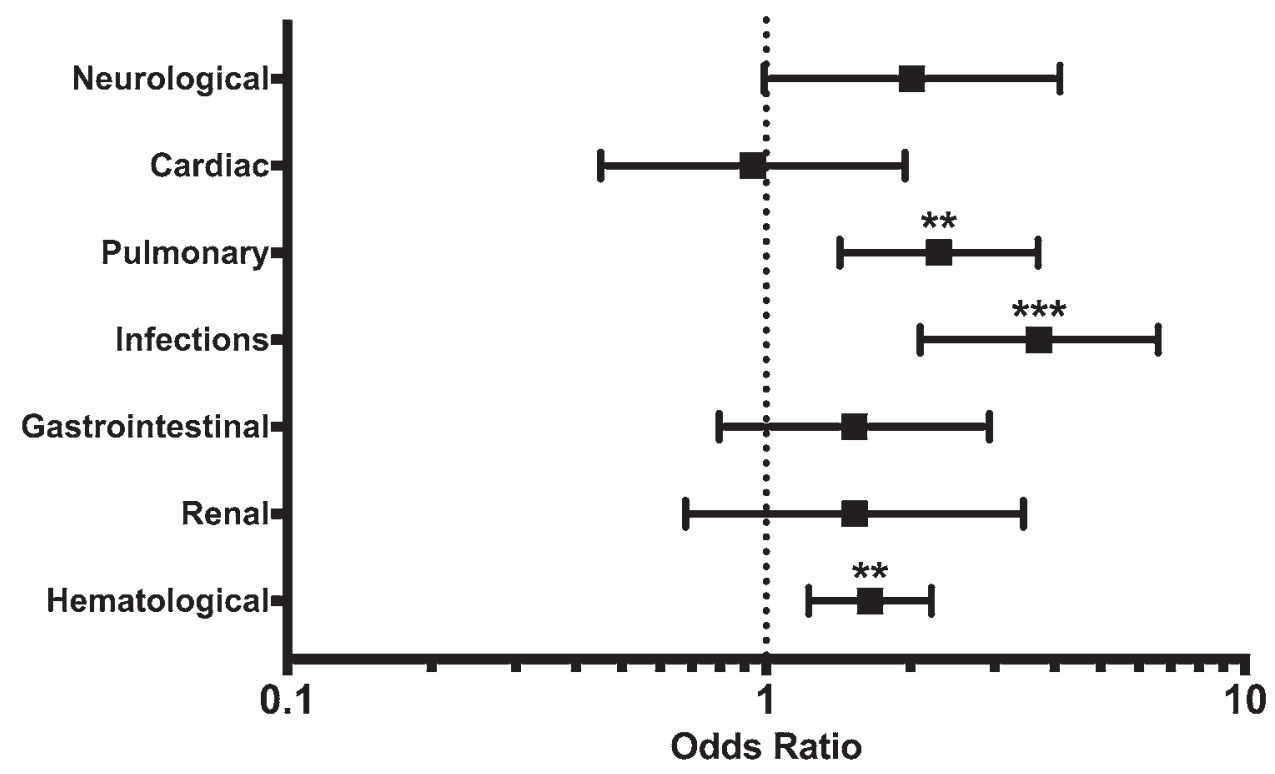

FIG. 2. Forest plot depicting the impact of OUD status on the odds of organ system complications following lumbar fusion procedures. Odds ratios obtained through univariate logistic regressions. ${ }^{* *} p<0.01 ;{ }^{* *} p<0.0001$.

$<0.0001)$ and higher total charges for their hospital stay $(\mathrm{p}=0.0076)$. This analysis also indicated that this patient population experienced significantly increased odds of a nonhome discharge after their hospital stay $(\mathrm{p}<0.0001)$, with $25.39 \%$ of patients discharged not to home compared with $15.63 \%$ of patients in the population without an OUD.

Multiple regression analyses were performed for each procedure type to better examine how OUDs impact

TABLE 4. Univariate regression models evaluating the impact of OUD status on certain surgical complications across organ systems

\begin{tabular}{|c|c|c|c|c|}
\hline & \multicolumn{2}{|c|}{ OUD Status* } & \multirow[b]{2}{*}{ OR $(95 \% \mathrm{Cl})$} & \multirow[b]{2}{*}{$\mathrm{p}$ Value } \\
\hline & $\operatorname{LDD}(n=138,715)$ & LDD + OUD $(n=1280)$ & & \\
\hline Myocardial infarction & $230(0.17)$ & $0(0)$ & NA & NA \\
\hline Cardiac arrest & $575(0.41)$ & $10(0.78)$ & $1.892(0.452-7.925)$ & 0.3828 \\
\hline Renal failure & $2520(1.82)$ & $35(2.73)$ & $1.519(0.674-3.423)$ & 0.3127 \\
\hline Pneumonia & $1080(0.78)$ & $30(2.34)$ & $3.059(1.285-7.283)$ & 0.0115 \\
\hline Deep venous thrombosis & $160(0.12)$ & $0(0)$ & NA & NA \\
\hline Venous thromboembolism & $795(0.57)$ & $30(2.34)$ & 4.165 (1.851-9.372) & 0.0006 \\
\hline Acute respiratory failure & $685(0.49)$ & $10(0.78)$ & $1.587(0.383-6.571)$ & 0.5243 \\
\hline Pulmonary embolism & $330(0.24)$ & $15(1.17)$ & $4.973(1.499-16.500)$ & 0.0088 \\
\hline Postop anemia & $19,120(13.78)$ & $305(23.83)$ & $1.957(1.406-2.722)$ & $<0.0001$ \\
\hline Oliguria/anuria & $155(0.11)$ & $0(0)$ & NA & NA \\
\hline Urinary complication & $910(0.66)$ & $10(0.78)$ & $1.193(0.289-4.919)$ & 0.8076 \\
\hline Wound dehiscence & $65(0.05)$ & $0(0)$ & NA & NA \\
\hline Wound infection & $175(0.13)$ & $15(1.17)$ & $9.387(3.357-26.249)$ & $<0.0001$ \\
\hline Systemic inflammatory response syndrome & $170(0.12)$ & $15(1.17)$ & $9.664(2.846-32.810)$ & $<0.0001$ \\
\hline Severe sepsis & $140(0.10)$ & $5(0.39)$ & $3.882(0.516-29.221)$ & 0.1878 \\
\hline Septic shock & $45(0.03)$ & $0(0)$ & NA & NA \\
\hline Urinary tract infection & $2770(2.00)$ & $65(5.08)$ & $2.626(1.407-4.901)$ & 0.0024 \\
\hline Sodium disturbance complication & $4480(3.23)$ & $40(3.13)$ & $0.967(0.478-1.955)$ & 0.9257 \\
\hline Tracheostomy & $30(0.02)$ & $5(0.39)$ & $18.130(2.130-154.300)$ & 0.0080 \\
\hline Gastrojejunostomy & $20(0.01)$ & $0(0)$ & NA & NA \\
\hline
\end{tabular}

$p<0.05$ was used as a threshold for statistical significance.

${ }^{*}$ Values given as weighted count (\%). 
TABLE 5. Univariate regression models evaluating the impact of OUD status on resource utilization and secondary clinical outcomes

\begin{tabular}{|c|c|c|c|}
\hline & \multicolumn{2}{|c|}{ OUD Status } & \multirow[b]{2}{*}{ p Value } \\
\hline & LDD & LDD + OUD & \\
\hline Mean length of stay, days & $n=138,715$ & $n=1280$ & \\
\hline Mean $(95 \% \mathrm{Cl})$ & $3.465(3.407-3.522)$ & $4.977(4.499-5.454)$ & $<0.0001$ \\
\hline SEM & 0.029 & 0.244 & \\
\hline Nonhome discharge & $n=138,715$ & $n=1280$ & \\
\hline Weighted count (\%) & $21,680(15.63)$ & $325(25.39)$ & $<0.0001$ \\
\hline OR $(95 \% \mathrm{Cl})$ & \multicolumn{2}{|c|}{$1.837(1.361-2.481)$} & \\
\hline Total charges & $n=137,630$ & $n=1270$ & \\
\hline Mean $(95 \% \mathrm{Cl})$ & $120,664(117,458-123,870)$ & $150,895(128,511-173,280)$ & 0.0076 \\
\hline SEM & 1635 & 11,416 & \\
\hline
\end{tabular}

$p<0.05$ was used as a threshold for statistical significance.

large-scale primary and secondary clinical outcomes in these patient populations while controlling for important demographic and comorbidity variables (Table 6). The models in these analyses specifically examined the odds of a complication, a nonhome discharge, and an extended hospitalization, in addition to providing an estimate as to how much extra a patient with an OUD will be charged for the hospital stay for each procedure type. All of the models specifically controlled for demographic variables, including age, sex, race, primary payer status, hospital geographic division, and income quartile, as well as comorbidity variables, including alcohol use, deficiency anemia, anemia due to chronic blood loss, chronic lung disease, depression, hypertension, electrolyte and fluid disorders, obesity, peripheral vascular disease, psychosis, weight loss, and other neurological disorders.

In our model describing the effects of OUDs on ALIF outcomes, the odds of a nonhome discharge (OR 3.135, 95\% CI 1.620-6.064; $\mathrm{p}=0.0007)$ and extended hospitalization (OR 3.275, 95\% CI 1.770-6.060; $\mathrm{p}=0.0002$ ) were significantly higher for patients with an OUD, while the odds of experiencing a complication were not significant (OR 1.535, 95\% CI 0.692-3.406; $\mathrm{p}=0.2915)$. In contrast, our model for PLIF outcomes indicated that the odds of a complication (OR 2.436, 95\% CI 1.190-4.989; $\mathrm{p}=0.0149$ ) and an extended hospitalization (OR 1.941, 95\% CI 1.0183.698; $\mathrm{p}=0.0439$ ), but not nonhome discharge (OR 1.973, $95 \%$ CI $0.910-4.278 ; p=0.0853$ ), were significantly greater for patients with OUDs. Finally, in our model for LLIF outcomes, all of these variables differed significantly by OUD status. In addition, our models also indicated that patients with OUDs had significantly higher total charges for their hospital visits regardless of procedure technique. On average, this patient population was charged $\$ 17,926$ more for ALIFs $(\mathrm{p}=0.0054), \$ 25,440$ more for PLIFs $(\mathrm{p}<$ $0.0001)$, and $\$ 37,228$ more for LLIFs ( $p<0.0001)$.

\section{Discussion}

Opioid use in the United States has markedly increased over the past 2 decades as prescription opioid therapies are one of the most common treatments for chronic back pain. Numerous studies have suggested that postoperative outcomes may be improved by reducing opioid analgesic use., ${ }^{9,22,35}$ As such, ERAS protocols often emphasize reduction of opioid analgesics. However, patients with OUDs display problematic patterns of opioid consumption and are particularly difficult to manage with traditional ERAS

\begin{tabular}{|c|c|c|c|}
\hline Outcome & OR $(95 \% \mathrm{Cl})$ & Estimate (SE) & $p$ Value \\
\hline \multicolumn{4}{|l|}{ ALIF } \\
\hline Complication & $1.535(0.692-3.406)$ & & 0.2915 \\
\hline $\begin{array}{l}\text { Nonhome } \\
\text { discharge }\end{array}$ & $3.135(1.620-6.064)$ & & 0.0007 \\
\hline $\begin{array}{l}\text { Extended hos- } \\
\text { pitalization }\end{array}$ & $3.275(1.770-6.060)$ & & 0.0002 \\
\hline Total charges & & $\$ 17,926(6428)$ & 0.0054 \\
\hline \multicolumn{4}{|l|}{ PLIF } \\
\hline Complication & $2.436(1.190-4.989)$ & & 0.0149 \\
\hline $\begin{array}{l}\text { Nonhome } \\
\text { discharge }\end{array}$ & $1.973(0.910-4.278)$ & & 0.0853 \\
\hline $\begin{array}{l}\text { Extended hos- } \\
\text { pitalization }\end{array}$ & $1.941(1.018-3.698)$ & & 0.0439 \\
\hline Total charges & & $\$ 25,440(248)$ & $<0.0001$ \\
\hline \multicolumn{4}{|l|}{ LLIF } \\
\hline Complication & $1.668(1.007-2.763)$ & & 0.0471 \\
\hline $\begin{array}{l}\text { Nonhome } \\
\text { discharge }\end{array}$ & $2.206(1.317-3.695)$ & & 0.0027 \\
\hline $\begin{array}{l}\text { Extended hos- } \\
\text { pitalization }\end{array}$ & $2.446(1.558-3.839)$ & & 0.0001 \\
\hline Total charges & & $\$ 37,228(3886)$ & $<0.0001$ \\
\hline
\end{tabular}

Models control for demographic variables, such as age, sex, race, primary payer status, hospital census division, and income quartile, as well as comorbidities, including alcohol use, deficiency anemia, anemia due to chronic blood loss, chronic lung disease, depression, hypertension, electrolyte and fluid disorders, obesity, peripheral vascular disease, psychosis, weight loss, and other neurological disorders. $p<0.05$ was used as a threshold for statistical significance. 
protocols. More focused ERAS systems should be developed for OUD patients and must account for the unique risks and adverse outcomes faced by this population during their hospitalizations. In this study, we identified unique risk and outcome profiles that pertain to OUD patients that have not previously been discussed in the literature. This has limited the ability of ERAS protocols to provide optimal care in this population.

\section{Demographics and Comorbidities}

We observed several important differences between the demographics of the OUD and non-OUD patient populations. First, these groups showed statistically significant differences in the distributions of their ages, primary insurance payer, median income by ZIP code, discharge disposition, geographic hospital division, APR-DRG risk of mortality and severity of illness scores, and frequency of surgically relevant comorbidities such as alcohol abuse, deficiency anemia, arthritis, anemia due to chronic blood loss, chronic lung disease, depression, hypertension, fluid and electrolyte disorders, obesity, peripheral vascular disease, psychosis, and weight loss. Recognizing that these observed demographic differences could significantly impact measures of perioperative outcome and resource utilization, we constructed multivariate logistic regression models that controlled for these variables to better characterize how OUD status interacts with key perioperative variables to impact clinical outcome by the surgical approach taken to treat LDD.

\section{Complications}

Preoperative opioid use is known to be associated with increased rates of complication after a variety of surgical procedures. ${ }^{5,14,16}$ This study carefully assessed procedurerelated complications from a variety of perspectives. Patient populations differing in their OUD status were examined for postprocedural complications according to which surgically relevant complications occurred, which organ systems were involved, and which surgical approach was used for the procedure.

Studies have shown that patients with chronic opioid use may have an increased risk of wound complications postoperatively. ${ }^{14}$ For example, systemic opioid levels have been associated with decreased angiogenesis and fewer myofibroblasts and macrophages at the wound site, which ultimately impairs wound healing..$^{15,20,21}$ Importantly, results from this present study provide further support to this notion on an even larger scale. Based on our univariate regression models, we found that patients with OUDs were significantly more likely to experience wound infections after their procedures than patients without OUDs.

When examined from an organ systems perspective, we found that patients with OUDs had significantly higher odds of experiencing pulmonary, hematological, and infectious complications perioperatively (Fig. 2). Previous work has found that chronic opioid use is associated with higher levels of infectious complications, ${ }^{2}$ such as pneumonia, particularly in elderly patients.? This may be due to the broader immunosuppressive effects that some opioid medications exert by directly acting on the mu-opioid receptors found on immune cells as well as by indirectly affecting glucocorticoid levels by acting on the sympathetic nervous system. ${ }^{29,30}$ In this study, we observed that OUD patients experienced significantly higher odds of infectious complications, and, more specifically, pneumonia and urinary tract infections.

Recent studies have also found that patients with OUDs have an increased risk of developing pulmonary complications, including the need for extended mechanical intubation or reintubation. ${ }^{31}$ In this study, OUD patients had 2.3-fold higher odds of developing pulmonary complications than non-OUD patients and faced higher odds of experiencing pneumonia and pulmonary embolism, but not acute respiratory failure. Taken together, our results are consistent with those previously reported in the literature, suggesting that OUD patients face higher odds of pulmonary complications postoperatively.

When examining the complication profiles of patients based on their procedure type, we found differences in the odds of complication for OUD patients depending on surgical approach. Results from our multivariate analysis suggest that, after controlling for demographic and comorbidity variables, the odds of complication for OUD patients were only significant for PLIF and LLIF (Table 6). Accordingly, previous studies described differences in the types and frequencies of complications after lumbar fusion procedures that depend on the surgical approach. ${ }^{8}$ However, there is debate in the literature comparing the complication rates across different lumbar fusion approaches. Smaller, single-institution studies have found that an anterior approach offers a lower incidence of complication rates, $, 27,32$ while large cohort analyses have found that patients with anterior lumbar spinal surgery have higher rates of postoperative complications and pain medication usage than patients who undergo posterior lumbar surgery, independent of opioid use status. ${ }^{13,23}$

\section{Mortality}

We did not observe any significant increases in the odds of mortality for OUD patients in this study. Previous studies have identified age, sex, and certain comorbidities as risk factors that are most often associated with mortality

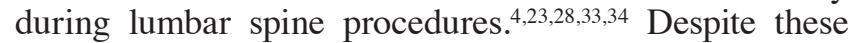
reported risk factors, it is also recognized that mortality rates in lumbar spine surgeries are historically low overall, with one large-scale study estimating an overall mortality rate of $0.13 \%$ across all lumbar surgical patients in the United States between 2003 and 2012. ${ }^{26}$ This same study identified older age, black race, male sex, liver disease, and congestive heart failure to be significantly associated with increased mortality rates. ${ }^{26}$ The OUD patients in this study tended to be younger and were not significantly different from non-OUD patients regarding sex, race, or congestive heart failure status, which may partially explain why mortality risk was not increased in this study.

\section{Resource Utilization and Secondary Outcomes}

Preoperative opioid use has been shown to independently influence costs, length of stay, and complication rates in surgical patients. ${ }^{5,14}$ Previous studies have also shown that patients with greater levels of preoperative opioid use also tend to have increased pain and pain perception compared 
with opioid-naive populations. ${ }^{10,12}$ Dagal et al. looked at a unique patient population in which more than half of the patients were on chronic opioid therapy. ${ }^{6}$ In this population, they developed a perioperative enhanced recovery care pathway for major spine surgery to improve postoperative outcomes in patients and found that patients with chronic opioid use prior to surgery reported more time in severe pain. In separate studies, there is increasing evidence that perioperative opioid consumption is associated with increased acute pain after surgeries and increased postoperative opioid use prior to discharge. ${ }^{10,25}$ This, in turn, can lead to prolonged hospitalizations, higher total costs, and increased readmission rates. ${ }^{11}$ Accordingly, in the present study, patients with OUDs tended to perform more poorly in terms of resource utilization metrics, including extended hospitalization, nonhome discharge, and higher total costs of hospitalization. As such, the development of a more individualized ERAS model for this patient population may allow for novel cost-saving and resource utilization measures that have been previously unexplored.

\section{Limitations}

This study was inherently limited by its retrospective design, which makes it challenging to examine temporal relationships and makes selection bias a potential concern. The NIS database also possesses certain intrinsic limitations. First, NIS data are encoded by ICD-9-CM codes, which means that certain measures not represented by an ICD-9-CM code could not be examined. These may include various measures of opioid use and pain. Of note, it was not possible to stratify opioid-using patients by their daily morphine equivalent doses, track patients over time, or assess other meaningful variables such as the level and extent of lumbar compression, blood loss, surgery duration, mobilization time, or anesthesia. Additionally, because the NIS does not track mortality outside the hospital, patient mortality may not be fully represented. Rare conditions may also not reflect their true value in the population due to the study's relatively short time window. Finally, these data represent cases performed under various circumstances, with individual-level differences in surgical approach. While this introduces a certain degree of heterogeneity in the underlying data set, it also perhaps improves the generalizability to practices across the nation given the smaller influences of the specific practices and policies of individual surgical teams.

\section{Conclusions}

This is the first study to characterize the outcome profiles for lumbar fusion procedures in patients with OUDs on a national level as a step toward developing new ERAS protocols in spine surgery for this unique patient population. Patients face particularly difficult recoveries after spine surgery, and OUD patients are an especially vulnerable population in the perioperative period for whom traditional ERAS protocols may not be the most effective. Developing more effective ERAS protocols for OUD patients will require a more detailed understanding of the unique problems this population may face in the perioperative period. This study examined how OUD status influenced primary outcomes, such as complications and mortality, as well as secondary outcomes and resource utilization metrics following a variety of lumbar fusion procedures.

Taken together, the results from this study suggest that OUD status is an important determinant of primary and secondary clinical outcomes after lumbar fusion procedures and poses certain perioperative risks that physicians, surgeons, and policymakers must be aware of when designing and implementing new enhanced recovery protocols to more optimally care for this patient population. These results warrant prospective studies to further the development of better ERAS programs that may improve patient care and reduce cost burden.

\section{References}

1. Armaghani SJ, Lee DS, Bible JE, Archer KR, Shau DN, Kay $\mathrm{H}$, et al: Preoperative opioid use and its association with perioperative opioid demand and postoperative opioid independence in patients undergoing spine surgery. Spine (Phila Pa 1976) 39:E1524-E1530, 2014

2. Baldini A, Von Korff M, Lin EH: A review of potential adverse effects of long-term opioid therapy: a practitioner's guide. Prim Care Companion CNS Disord 14:PCC.11m01326, 2012

3. Campbell PG, Yadla S, Malone J, Maltenfort MG, Harrop JS, Sharan AD, et al: Complications related to instrumentation in spine surgery: a prospective analysis. Neurosurg Focus 31(4):E10, 2011

4. Cloyd JM, Acosta FL Jr, Cloyd C, Ames CP: Effects of age on perioperative complications of extensive multilevel thoracolumbar spinal fusion surgery. J Neurosurg Spine 12:402408, 2010

5. Cron DC, Englesbe MJ, Bolton CJ, Joseph MT, Carrier KL, Moser SE, et al: Preoperative opioid use is independently associated with increased costs and worse outcomes after major abdominal surgery. Ann Surg 265:695-701, 2017

6. Dagal A, Lesnik I, Bentov N, Dong J, Qiu Q, Hayes K, et al: Using a perioperative enhanced recovery care pathway for major spine surgery (PERMS) to improve pain management \& patient-reported outcomes. J Pain 17:S89, 2016

7. Dublin S, Walker RL, Jackson ML, Nelson JC, Weiss NS, Von Korff M, et al: Use of opioids or benzodiazepines and risk of pneumonia in older adults: a population-based casecontrol study. J Am Geriatr Soc 59:1899-1907, 2011

8. Elsamadicy AA, Adogwa O, Behrens S, Sergesketter A, Chen A, Mehta AI, et al: Impact of surgical approach on complication rates after elective spinal fusion ( $\geq 3$ levels) for adult spine deformity. J Spine Surg 3:31-37, 2017

9. Farag E, Ghobrial M, Sessler DI, Dalton JE, Liu J, Lee JH, et al: Effect of perioperative intravenous lidocaine administration on pain, opioid consumption, and quality of life after complex spine surgery. Anesthesiology 119:932-940, 2013

10. Fletcher D, Martinez V: Opioid-induced hyperalgesia in patients after surgery: a systematic review and a meta-analysis. Br J Anaesth 112:991-1004, 2014

11. Gupta A, Nizamuddin J, Elmofty D, Nizamuddin SL, Tung A, Minhaj M, et al: Opioid abuse or dependence increases 30-day readmission rates after major operating room procedures: a National Readmissions Database study. Anesthesiology 128:880-890, 2018

12. Hina N, Fletcher D, Poindessous-Jazat F, Martinez V: Hyperalgesia induced by low-dose opioid treatment before orthopaedic surgery: an observational case-control study. Eur J Anaesthesiol 32:255-261, 2015

13. Huang KT, Hazzard M, Thomas S, Chagoya G, Berg RW, Adogwa O, et al: Differences in the outcomes of anterior versus posterior interbody fusion surgery of the lumbar spine: 
a propensity score-controlled cohort analysis of $10,941 \mathrm{pa}-$ tients. J Clin Neurosci 22:848-853, 2015

14. Jain N, Phillips FM, Weaver T, Khan SN: Preoperative chronic opioid therapy: a risk factor for complications, readmission, continued opioid use and increased costs after oneand two-level posterior lumbar fusion. Spine (Phila Pa 1976) 43:1331-1338, 2018

15. Lam CF, Chang PJ, Huang YS, Sung YH, Huang CC, Lin $\mathrm{MW}$, et al: Prolonged use of high-dose morphine impairs angiogenesis and mobilization of endothelial progenitor cells in mice. Anesth Analg 107:686-692, 2008

16. Lee D, Armaghani S, Archer KR, Bible J, Shau D, Kay H, et al: Preoperative opioid use as a predictor of adverse postoperative self-reported outcomes in patients undergoing spine surgery. J Bone Joint Surg Am 96:e89, 2014

17. Ljungqvist O, Scott M, Fearon KC: Enhanced Recovery After Surgery: a review. JAMA Surg 152:292-298, 2017

18. Lønne G, Fritzell P, Hägg O, Nordvall D, Gerdhem P, Lagerbäck T, et al: Lumbar spinal stenosis: comparison of surgical practice variation and clinical outcome in three national spine registries. Spine J 19:41-49, 2019

19. Martin B, Mirza SK, Spina N, Spiker WR, Lawrence B, Brodke DS: Trends in lumbar fusion procedure rates and associated hospital costs for degenerative spinal diseases in the United States, 2004-2015. Spine (Phila Pa 1976) [epub ahead of print], 2018

20. Martin JL, Charboneau R, Barke RA, Roy S: Chronic morphine treatment inhibits LPS-induced angiogenesis: implications in wound healing. Cell Immunol 265:139-145, 2010

21. Martin JL, Koodie L, Krishnan AG, Charboneau R, Barke RA, Roy S: Chronic morphine administration delays wound healing by inhibiting immune cell recruitment to the wound site. Am J Pathol 176:786-799, 2010

22. Mathiesen O, Dahl B, Thomsen BA, Kitter B, Sonne N, Dahl JB, et al: A comprehensive multimodal pain treatment reduces opioid consumption after multilevel spine surgery. Eur Spine J 22:2089-2096, 2013

23. Memtsoudis SG, Vougioukas VI, Ma Y, Gaber-Baylis LK, Girardi FP: Perioperative morbidity and mortality after anterior, posterior, and anterior/posterior spine fusion surgery. Spine (Phila Pa 1976) 36:1867-1877, 2011

24. Menendez ME, Ring D, Bateman BT: Preoperative opioid misuse is associated with increased morbidity and mortality after elective orthopaedic surgery. Clin Orthop Relat Res 473:2402-2412, 2015

25. Myers J, Compton P: Addressing the potential for perioperative relapse in those recovering from opioid use disorder. Pain Med 19:1908-1915, 2018

26. Poorman GW, Moon JY, Wang C, Horn SR, Beaubrun BM, Bono OJ, et al: Rates of mortality in lumbar spine surgery and factors associated with its occurrence over a 10 -year period: a study of 803,949 patients in the Nationwide Inpatient Sample. Int J Spine Surg 12:617-623, 2018

27. Pradhan BB, Nassar JA, Delamarter RB, Wang JC: Singlelevel lumbar spine fusion: a comparison of anterior and posterior approaches. J Spinal Disord Tech 15:355-361, 2002

28. Pumberger M, Chiu YL, Ma Y, Girardi FP, Vougioukas V, Memtsoudis SG: Perioperative mortality after lumbar spinal fusion surgery: an analysis of epidemiology and risk factors. Eur Spine J 21:1633-1639, 2012
29. Sacerdote P: Opioid-induced immunosuppression. Curr Opin Support Palliat Care 2:14-18, 2008

30. Sacerdote P, Franchi S, Panerai AE: Non-analgesic effects of opioids: mechanisms and potential clinical relevance of opioid-induced immunodepression. Curr Pharm Des 18:6034-6042, 2012

31. Sayal P, Bateman BT, Menendez M, Eikermann M, Ladha KS: Opioid use disorders and the risk of postoperative pulmonary complications. Anesth Analg 127:767-774, 2018

32. Scaduto AA, Gamradt SC, Yu WD, Huang J, Delamarter RB, Wang JC: Perioperative complications of threaded cylindrical lumbar interbody fusion devices: anterior versus posterior approach. J Spinal Disord Tech 16:502-507, 2003

33. Schoenfeld AJ, Reamer EN, Wynkoop EI, Choi H, Bono CM: Does patient sex affect the rate of mortality and complications after spine surgery? A systematic review. Clin Orthop Relat Res 473:2479-2486, 2015

34. Smith JS, Saulle D, Chen CJ, Lenke LG, Polly DW Jr, Kasliwal MK, et al: Rates and causes of mortality associated with spine surgery based on 108,419 procedures: a review of the Scoliosis Research Society Morbidity and Mortality Database. Spine (Phila Pa 1976) 37:1975-1982, 2012

35. Wainwright TW, Immins T, Middleton RG: Enhanced recovery after surgery (ERAS) and its applicability for major spine surgery. Best Pract Res Clin Anaesthesiol 30:91-102, 2016

36. Ward EN, Quaye AN, Wilens TE: Opioid use disorders: perioperative management of a special population. Anesth Analg 127:539-547, 2018

37. Zywiel MG, Stroh DA, Lee SY, Bonutti PM, Mont MA: Chronic opioid use prior to total knee arthroplasty. J Bone Joint Surg Am 93:1988-1993, 2011

\section{Disclosures}

The authors report no conflict of interest concerning the materials or methods used in this study or the findings specified in this paper.

\section{Author Contributions}

Conception and design: Caridi, Martini. Acquisition of data: Caridi, Martini, Deutsch. Analysis and interpretation of data: all authors. Drafting the article: Martini, Nistal. Critically revising the article: Caridi, Martini, Nistal. Reviewed submitted version of manuscript: Caridi, Martini, Nistal. Statistical analysis: Martini, Deutsch. Study supervision: Caridi, Martini.

\section{Correspondence}

John M. Caridi: Icahn School of Medicine at Mount Sinai, New York, NY.john.caridi@mountsinai.org. 